> L'acide myristique est un acide gras saturé à 14 carbones qui est majoritairement retrouvé dans la matière grasse laitière. Dans les pays industrialisés, sa consommation excessive est corrélée à une hausse du cholestérol plasmatique et à une augmentation de la mortalité due à des maladies cardiovasculaires. Néanmoins, l'une des caractéristiques de cet acide gras est sa capacité à acyler les protéines : la myristoylation. Cet article expose différents exemples de régulations cellulaires où l'intervention de l'acide myristique est avérée. La concentration en acide myristique et le niveau de myristoylation des protéines semblent donc être des facteurs de régulation de nombreux mécanismes cellulaires. <

\section{Acide myristique : nouvelles fonctions de régulation et de signalisation}

Erwan Beauchamp, Vincent Rioux, Philippe Legrand

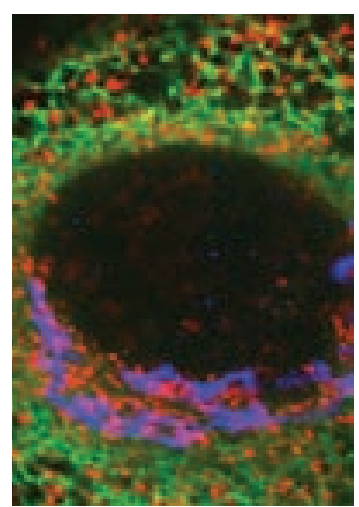

Laboratoire de BiochimieNutrition Humaine, Agrocampus Rennes-INRA USC 2012, 65 , rue de Saint-Brieuc, 35042 Rennes Cedex, France. Vincent.Rioux@ agrocampus-rennes.fr
Parmi les acides gras saturés les plus consommés dans l'alimentation humaine, l'acide myristique (acide saturé linéaire à 14 carbones, $\mathrm{CH}_{3}-\left[\mathrm{CH}_{2}\right]_{12}-\mathrm{COOH}$, $\mathrm{C} 14: 0)$ arrive en troisième position, loin derrière l'acide palmitique $\left(\mathrm{CH}_{3}-\left[\mathrm{CH}_{2}\right]_{14}-\mathrm{COOH}, \mathrm{Cl} 6: 0\right)$ et l'acide stéarique $\left(\mathrm{CH}_{3}-\left[\mathrm{CH}_{2}\right]_{16}-\mathrm{COOH}, \mathrm{Cl} 18: 0\right)$ [1]. Présent en quantité importante dans la matière grasse laitière (il représente environ $10 \%$ des acides gras), l'acide myristique occupe majoritairement la position $s n-2^{1}$ [2] sur les triglycérides, ce qui lui assure une absorption intestinale efficace sous forme de 2-monoglycéride. Dans la cellule animale, l'acide myristique est relativement rare et représente en moyenne $1 \%$ des acides gras. Sa biosynthèse endogène faible (quelques centaines de $\mu \mathrm{g}$ dans le foie) rend son origine alimentaire quantitativement prépondérante (entre 4 et $8 \mathrm{~g} /$ jour) [3]. Lorsqu'il est ajouté à des hépatocytes en culture, l'acide myristique est majoritairement incorporé dans les lipides cellulaires ( $65 \%$ du C14:0 initial) mais est aussi largement $\beta$-oxydé (30\%) (Figure 1) [4].

La description des rôles physiologiques de l'acide myristique a longtemps été restreinte à sa responsabilité dans la hausse du cholestérol plasmatique chez l'homme et l'animal, quand il est apporté en excès dans

${ }^{1}$ Sn dénote les atomes de carbone. l'alimentation [5], ce qui explique sa mauvaise réputation nutritionnelle. Ces résultats, bien que remis en cause depuis que les études nutritionnelles explorent l'impact des doses raisonnables d'acides gras saturés totaux et d'acide myristique en particulier $[6,7]$, ont largement contribué à masquer les autres fonctions biologiques importantes de cet acide gras ainsi que les bases moléculaires de ces fonctions.

\section{Acide myristique et $\mathrm{N}$-myristoyltransférases}

Parmi les acides gras saturés (et les insaturés), seul l'acide myristique, après activation en myristoyl-CoA, possède la capacité de former irréversiblement une liaison amide (Figures 1 et 2) avec des protéines possédant une extrémité amino-terminale débutant obligatoirement par une Glycine, mais dont il est difficile d'extraire une séquence consensuelle en acides aminés [8]. Cette réaction, appelée myristoylation $\mathrm{N}$-terminale, est catalysée par la N-myristoyltransférase (NMT), produit de deux gènes très homologues identifiés chez les animaux et codant pour deux isoformes (NMT1 et NMT2) $[9,10]$. La majorité des études s'est focalisée sur la première et la plus active des deux isoformes [11]. Celle-ci possède une forte spécificité pour son substrat myristoyl-CoA [12] (le $\mathrm{Km}$ apparent varie entre $\mathrm{l}$ et $100 \mu \mathrm{m}$ en fonction des conditions expérimentales, de l'origine de l'enzyme, du type de co-substrat protéique...), ce qui s'explique par la structure de son site catalytique [13]. La concentration 


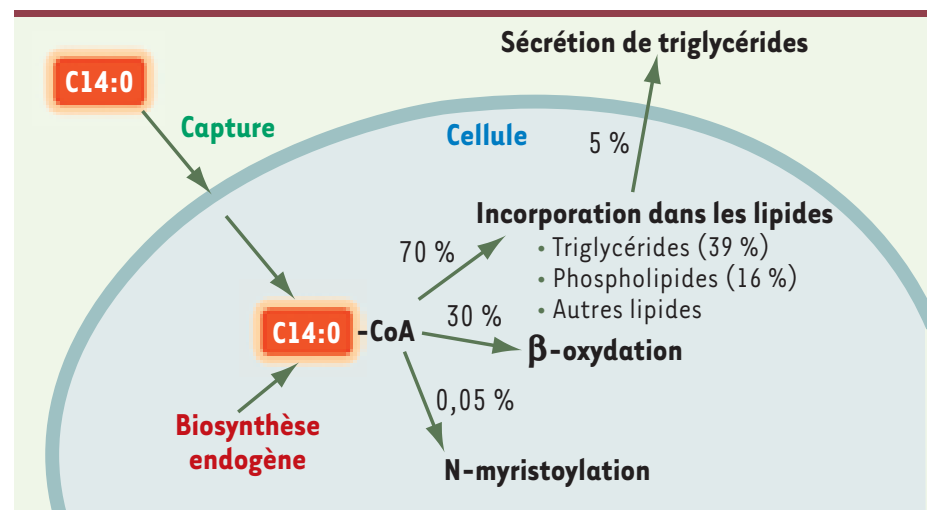

cellulaire en myristoyl-CoA disponible pour l'acylation des protéines est très faible, de l'ordre de $5 \mathrm{nM}$ [14]. Seulement 0,05 \% de l'acide myristique initial (Figure 1) ajouté à des hépatocytes en culture est utilisé pour la myristoylation N-terminale [15]. La NMT semble capable d'utiliser aussi bien l'acide myristique exogène [8] que l'acide myristique issu de la biosynthèse endogène, quand celui-ci est en concentration suffisante, après élongation à partir d'acide laurique ou rétroconversion de l'acide palmitique par exemple $[15,16]$. Dans la rétine, tissu ayant une composition spécifique en acides gras, la biodisponibilité du substrat acide gras semble prépondérante pour l'acylation des protéines. En effet, 2 acides gras rares insaturés à 14 carbones (C14:1 n-9 et C14:2 n-6) [17], dont la concentration sous forme d'acyl-CoA est plus importante dans la rétine que dans les autres tissus, peuvent remplacer l'acide myristique comme substrat de la NMT [18]. La concentration cellulaire en myristoyl-CoA est donc un régulateur important du niveau de myristoylation dans la cellule.

La myristoylation est majoritairement une réaction co-traductionnelle. La glycine amino-terminale du substrat protéique en cours de traduction est exposée à la NMT après action de la méthionyl aminopeptidase qui retire la méthionine initiatrice. Récemment, une myristoylation post-traductionnelle a été mise en évidence dans des cellules en apoptose, après clivage du substrat protéique par des caspases, qui expose alors un site interne de myristoylation [19]. La libération du site de myristoylation de ces protéines est soumise à régulation et dépend de l'état physiologique de la cellule.

\section{Protéines myristoylées et fonctions de la myristoylation}

Les protéines myristoylées connues sont des facteurs clés de la signalisation intracellulaire (sous-unité $\alpha$ des protéines G, Myristoylated alanine-rich C-kinase substrate ou MARCKS, un substrat ubiquitaire de la Protein kinase $(. .$.$) , des oncogènes, mais aussi des suppresseurs$ de tumeur, des protéines virales de structure mais aussi des protéines eucaryotes communes (NADH-cytochrome b5 réductase). Nous ne détaillerons pas ici les régulations associées à la myristoylation qui sont bien documentés pour ces protéines. Une étude bioinformatique prédictive a estimé à $0,5 \%$ la proportion de protéines myristoylées dans le protéome humain [20]. Parmi les quelques 25000 protéines issues des gènes décrits après le séquençage du génome humain, une
Figure 1. Les voies du métabolisme de l'acide myristique dans la cellule. Après captage par la cellule ou biosynthèse endogène, le devenir métabolique de l'acide myristique se répartit entre incorporation dans les lipides, $\beta$-oxydation et autres réactions plus minoritaires comme la myristoylation $\mathrm{N}$-terminale.

centaine de protéines myristoylées a été identifiée à ce jour [21]. Il en reste donc encore quelques-unes à découvrir, y compris celles dont la myristoylation survient à une étape post-traductionnelle.

La myristoylation d'une protéine induit des modifications importantes pour la protéine myristoylée : ancrage à la membrane, interactions avec d'autres protéines, changement d'adressage subcellulaire (Figure 2). Par cette voie, l'acide myristique a donc un rôle spécifique de régulation de l'activité biologique des protéines myristoylées. Dans de nombreux modèles (levures, drosophile, souris), la délétion du gène codant pour la NMT a des conséquences importantes allant d'altérations du développement à la mort du modèle [22, 23].

La découverte de la myristoylation $\mathrm{N}$-terminale des protéines dans la cellule eucaryote animale et végétale, ainsi que chez les virus, a suscité un regain d'intérêt pour cet acide gras, et l'on découvre de nouvelles fonctions de régulation et de signalisation liées, directement ou non, à la myristoylation. Cette revue se limitera donc à développer quelques exemples récents impliquant l'acide myristique et/ou la myristoylation dans différentes régulations cellulaires.

\section{Acide myristique et régulation de la signalisation par la synthase de monoxyde d'azote des cellules endothéliales (eNOS)}

La synthase de monoxyde d'azote (NOS) permet la formation du radical libre, le monoxyde d'azote (NO), en catalysant l'oxydation de l'arginine en citrulline [24]. L'enzyme endothéliale (eNOS), présente au niveau de la paroi des vaisseaux sanguins, est myristoylée. Les premières études de localisation subcellulaire ont montré que l'enzyme est membranaire et plus spécialement située dans les cavéoles [25]. Des expériences de mutagenèse dirigée contre le site de myristoylation ont montré que dans ces mutants, l'adressage de la protéine vers la membrane ne se faisait plus et qu'elle restait alors exclusivement cytosolique [26]. La protéine eNOS est également palmitoylée : cette double acylation est nécessaire à son adressage vers la membrane, et la dépalmitoylation permet la régulation de cet adressage [27, 28]. La myristoylation est donc le déterminant majeur, mais pas unique, de l'ancrage d'eNOS dans la membrane. Cette localisation 


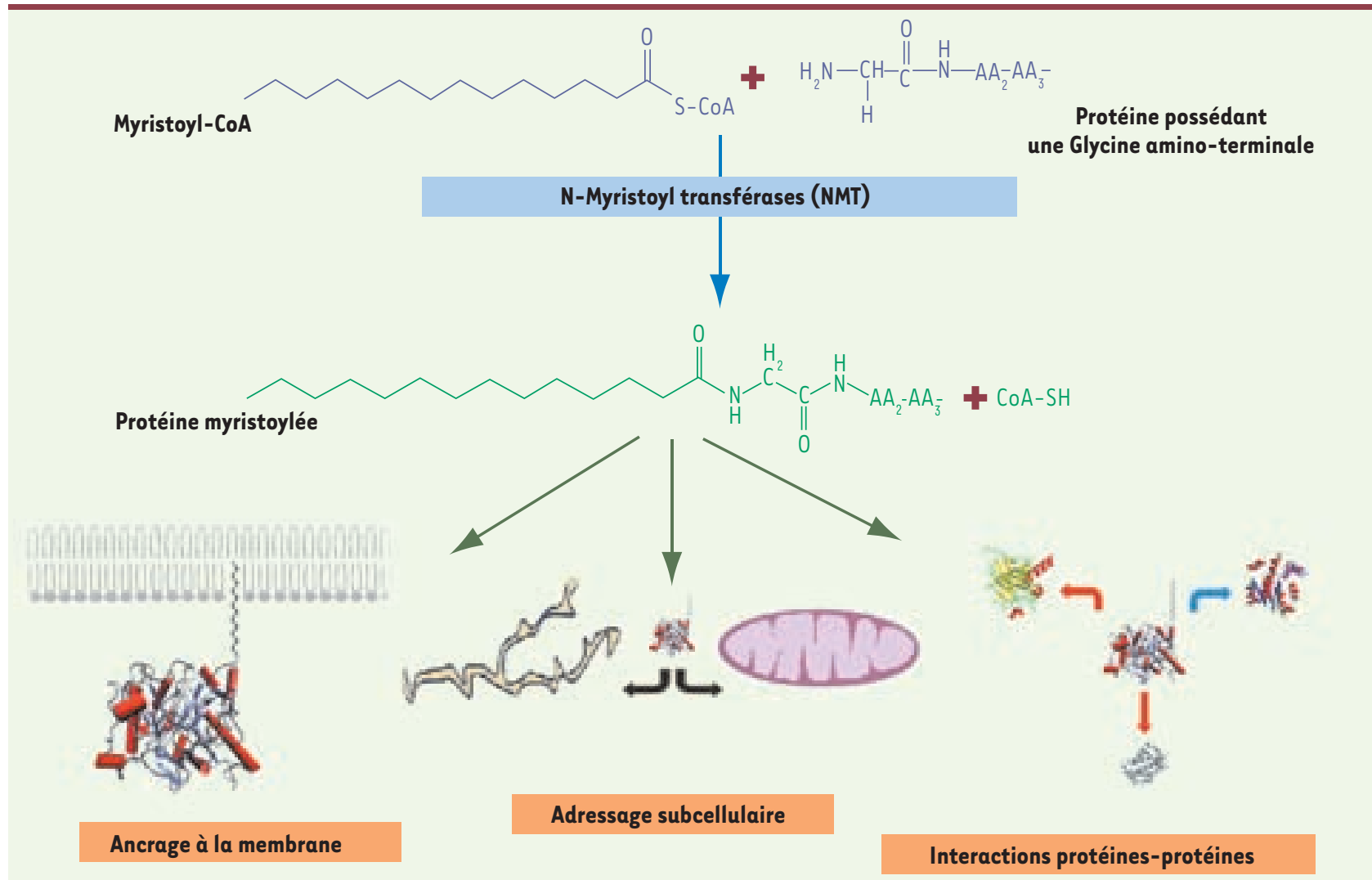

Figure 2. Myristoylation N-terminale des protéines et conséquences biologiques sur les protéines myristoylées. Les N-myristoyltransférases (NMT) catalysent la formation d'une liaison amide entre le myristoyl-CoA et une protéine possédant au moins une Glycine amino-terminale. L'acide myristique ainsi lié permet à la protéine soit de s'ancrer à la membrane, soit de changer d'adressage subcellulaire ou d'interagir avec d'autres protéines.

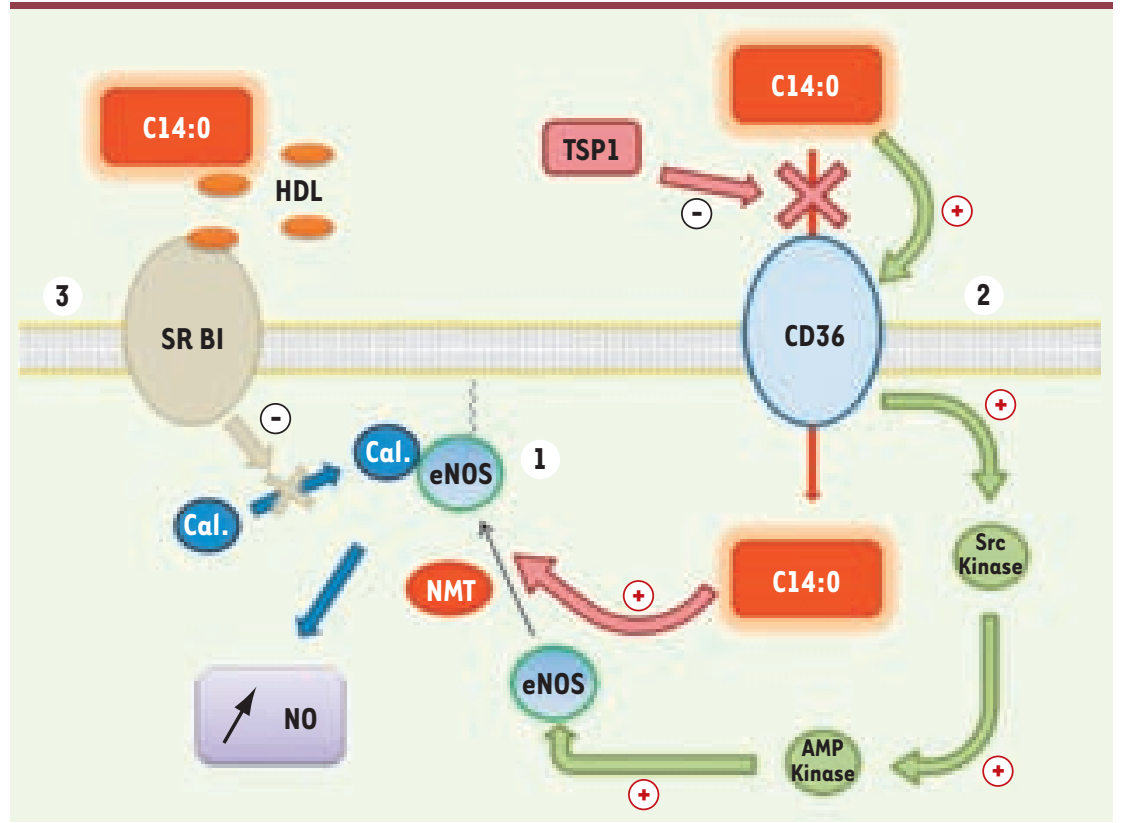

Figure 3. Mécanismes d'activation de la synthase de monoxyde d'azote endothéliale (eNOS) par l'acide myristique. 1. La synthase de monoxyde d'azote (NO) est myristoylée et alors adressée à la membrane plasmique [25]. Récemment, il a été montré que l'acide myristique pourrait interagir avec le récepteur CD36 et activerait eNOS via les Src et AMP kinases. L'acide myristique capté par le CD36 pourrait également être spécifiquement dédié à la myristoylation de eNOS. 2. Cette capture est inhibée par la thrombospondine-1 (TSPI). 3. En revanche, lorsqu'il est apporté sous forme estérifiée dans les HDL, l'acide myristique inhibe l'effet activateur des œstrogènes sur l'activité d'eNOS en inhibant l'association de l'enzyme avec la calmoduline (Cal). SR-BI : récepteur scavenger de classe $B$ et de type $I$.

membranaire pourrait permettre une meilleure libération extracellulaire et une action vasodilatatrice plus efficace du monoxyde d'azote.
Récemment, un autre niveau de régulation d'eNOS par l'acide myristique a été mis en évidence (Figure 3). Dans le modèle cellulaire endothélial, l'acide myristique active spécifiquement eNOS de manière dépendante 
du temps (quelques minutes), de la dose et de la présence d'AMPc [29]. L'acide myristique interagirait, sans myristoylation avérée, avec le récepteur «éboueur » CD36, lui aussi situé dans les cavéoles, et activerait une cascade de réactions [30]. Le récepteur CD36 pourrait activer I'AMP kinase, via la stimulation des Src kinases, qui activerait à son tour eNOS. Sans redémontrer l'effet spécifique du C14:0 via CD36 sur la production de $\mathrm{N} 0$, Isenberg et al. [31] suggèrent que l'inhibition du captage d'acide myristique par CD36, par l'ajout de thrombospondine 1 (un inhibiteur endogène de l'angiogenèse via $C D 36$ ) entraîne une inhibition de la production de monoxyde d'azote. Ils suggèrent également que l'acide myristique exogène capté via CD36 peut être spécifiquement orienté vers la myristoylation de certaines protéines. En effet, sur le même modèle, l'adressage de Fyn (une kinase de la famille Src) vers la membrane, après sa myristoylation, est stoppé si l'on inhibe le captage de l'acide myristique par CD36, même en ajoutant du C14:0 exogène au milieu de culture. Ces deux études démontrent que l'acide myristique libre peut avoir une action spécifique, à de faibles concentrations (entre 10 et $50 \mu \mathrm{M})$, dans la cellule endothéliale. Cependant, le mécanisme n'est pas totalement élucidé car ces deux études n'ont pas mesuré l'impact réel de la myristoylation sur l'activité d'eNOS en utilisant des mutants non myristoylables de l'enzyme, ni la spécificité d'orientation de l'acide myristique capté via CD36 vers la myristoylation d'eNOS.

De plus, il semble que l'acide myristique puisse avoir un rôle important dans l'action connue de l'œstradiol sur l'activité d'eNOS. En effet, il a été démontré que l'œstradiol, transporté par les HDL (high density lipoproteins), était capable d'activer spécifiquement eNOS [32]. Cependant, cette activation n'est plus retrouvée chez les patientes diabétiques. Or, comparées aux HDL témoins, la seule différence significative des HDL isolées chez les diabétiques est leur composition, puisqu'elles contiennent 3 à 4 fois plus d'acide myristique [33]. Des HDL issues de patients sains supplémentés en C14:0 ont un effet inhibiteur identique sur l'activité eNOS

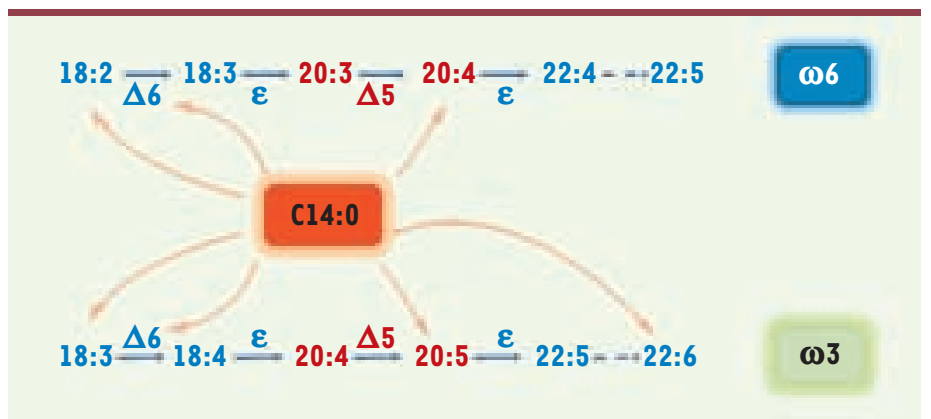

Figure 4. Régulations par l'acide myristique de la biosynthèse des acides gras hautement insaturés des familles $n-6$ et $n-3$ à partir de leurs précurseurs acide linoléique (C18:2 n-6) et acide $\alpha$-linolénique (C18:3n-3). In vitro, l'acide myristique augmente l'activité de la $\Delta 6$-désaturase qui catalyse la première étape de conversion des acides gras précurseurs ( $\Delta 6: \Delta 6$-désaturase, $\Delta 5: \Delta 5$ désaturase, $\varepsilon$ : élongase). In vivo chez le rat, l'acide myristique alimentaire augmente la concentration en acides linoléique et arachidonique (C20:4 n-6) de la famille $n-6$ dans le cerveau, et la concentration en acides $\alpha$-linolénique, eicosapentaénoique (c20:5n-3) et docosahexaénoique (C22:6n-3) dans le cerveau, le plasma et les érythrocytes.
(Figure 3). Cette nouvelle régulation par l'acide myristique semble passer par l'inhibition de l'association entre eNOS et la calmoduline (le récepteur majeur intracellulaire du calcium). Ces études démontrent l'importance du vecteur de transport (complexe albuminique ou $\mathrm{HDL}$ ) et de la forme d'apport (acide gras non estérifié ou triglycérides) de l'acide myristique pour la régulation d'eNOS.

\section{Acide myristique et régulation de la biodisponibilité des acides gras poly-insaturés}

Il est bien admis qu'une augmentation de la teneur cellulaire en acides gras poly-insaturés de la famille des $\omega 3$ (oméga 3), et notamment des dérivés les plus longs (acides eicosapentaénoïque ou EPA, et docosahexaénoïque ou DHA), est bénéfique pour la prévention des maladies cardio-vasculaires et plus généralement pour la santé humaine $[34,35]$. Des stratégies d'augmentation de la teneur en $\omega 3$ par des régimes enrichis en acide myristique ont été évaluées après l'observation de l'effet activateur de l'acide myristique sur la $\Delta 6$-désaturase [36]. Cette enzyme intervient dans la biosynthèse des acides gras poly-insaturés à très longues chaînes de la famille des $\omega 6$ et $\omega 3$, en introduisant une double liaison sur les précurseurs acides linoléique $(\mathrm{C} 18: 2 \mathrm{n}-6)$ et $\alpha$-linolénique (C18:3 n-3) (Figure 4). In vivo, chez le rat nourri pendant 2 mois par des régimes contenant des doses croissantes d'acide myristique, la concentration en précurseur acide $\alpha$-linolénique augmente dans tous les tissus de façon dose-dépendante, et les dérivés à très longues chaînes de la famille des $\omega 3$, dont l'EPA et le DHA, augmentent dans le cerveau et les globules rouges $[37,38]$. L'acide myristique et l'acide $\alpha$-linolénique sont tous deux des substrats de la $\beta$-oxydation. Une $\beta$-oxydation préférentielle de l'acide myristique semble épargner celle de l'acide $\alpha$-linolénique. Cet effet d'épargne du précurseur s'ajoute sans doute à l'effet activateur de l'acide myristique sur la biosynthèse des dérivés hautement insaturés. Chez l'homme, un régime assurant un apport modéré en acide myristique (1,2\% de l'énergie totale), comparé à un régime assurant un apport plus faible $(0,6 \%)$, augmente également les taux de DHA et d'EPA dans les phospholipides plasmatiques et le taux de DHA dans les esters de cholestérol plasmatiques [39]. Le mécanisme moléculaire à l'origine de l'activation par le C14:0 de la $\Delta 6$-désaturase dans le modèle cellulaire n'est pas identifié, mais l'hypothèse d'une myristoylation de l'enzyme, qui possède une Glycine amino-terminale, a été récemment écartée [40]. Une autre protéine du complexe $\Delta 6$-désaturant, la NADH-cytochrome b5 réductase, est en revanche myristoylée et son niveau de myristoylation pourrait donc être un paramètre de régulation [41]. 


\section{Acide myristique et régulation de la biosynthèse de novo du céramide}

Les sphingolipides constituent une classe importante de phospholipides et sont des composants essentiels des cellules eucaryotes. En plus de leur rôle structural dans la membrane plasmique, ils interviennent dans des processus cellulaires comme l'apoptose, la réponse au stress ou la croissance cellulaire. Récemment, une nouvelle protéine myristoylée intervenant dans le métabolisme de ces lipides a été découverte [40]. II s'agit de la dihydrocéramide $\Delta 4$-désaturase (DES) qui catalyse la dernière étape de la biosynthèse de novo du céramide (le précurseur de tous les sphingolipides) en introduisant une double liaison en position 4 sur le dihydrocéramide. Cette double liaison semble associée à des propriétés régulatrices du céramide, que ne possède pas son précurseur le dihydrocéramide, notamment sur l'apoptose [39]. En effet, le céramide peut former des canaux dans la membrane externe de la mitochondrie, permettant le relargage de petites protéines (notamment le cytochrome c) [42]. La myristoylation des deux isoformes de la dihydrocéramide $\triangle 4$-désaturase (DES1 et DES2) a été démontrée chez le rat. Cette acylation modifie les propriétés de l'enzyme puisque la mutation du site de myristoylation diminue de moitié l'activité désaturase de DESI (la forme la plus active) [40]. Les études actuelles tentent de déterminer si la myristoylation de DESI influe sur l'adressage subcellulaire de la protéine, notamment son adressage vers la mitochondrie (lieu de l'action potentielle pro-apoptotique du

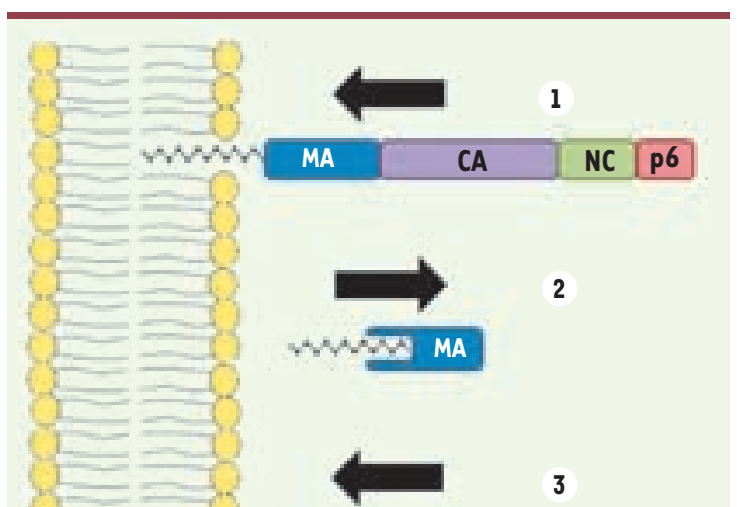

céramide) comme cela a déjà été observé pour la NADH cytochrome b5 réductase [41]. L'acide myristique pourrait donc, par le biais du métabolisme des sphingolipides, moduler l'apoptose.

\section{Acide myristique, myristoylation des protéines virales de structure et réplication des rétrovirus}

Au cours de la réplication des rétrovirus, comme celui de l'immunodéficience humaine $(\mathrm{VIH})$, le produit de l'expression du gène gag semble être le seul nécessaire à la formation des particules virales [43]. La protéine Gag est synthétisée sous la forme d'un précurseur polyprotéique Pr55gag [44] qui comprend plusieurs domaines dont un domaine matriciel (MA). Ce domaine est responsable de l'adressage des protéines virales vers la membrane plasmique. II est connu depuis plusieurs années que le domaine matriciel amino-terminal de Pr5 $55^{g a g}$ est myristoylé [45]. Des études récentes suggèrent que la protéine virale myristoylable Gag a une affinité différente pour les deux isoformes NMT1 et NMT2 présentes dans la cellule infectée mais ces résultats sont parfois discordants $[46,47]$. Cette myristoylation, associée à la présence d'un domaine polybasique, permet l'ancrage à la membrane du précurseur polyprotéique Pr $55^{g a g}$, étape nécessaire au clivage par les protéases virales qui libère alors une protéine MA myristoylée mature (Figure 5). Bien que myristoylée, la protéine MA mature isolée perd son affinité pour la membrane cellulaire (l'acide myristique est séquestré à l'intérieur de la protéine, c'est l'hypothèse du switch du C14:0) et devient majoritairement cytosolique [48]. Cependant, dès que la concentration de cette protéine augmente dans le cytosol au cours de l'infection virale, elle forme des trimères qui sont alors capables d'exposer à nouveau l'acide myristique, et de retourner s'ancrer dans la membrane, étape indispensable à l'assemblage de la matrice des particules virales (Figure 5).

Dans une telle situation d'infection virale, inhiber la myristoylation est donc une cible thérapeutique potentielle pour limiter la réplication et le cycle viral [49]. Ainsi, une inhibition de la myristoylation par des compétiteurs ( $\mathrm{C} 14: 1 \mathrm{n}-9$ et $\mathrm{Cl} 4: 2 \mathrm{n}-6)$ a montré une diminution du nombre de particules virales [50]. Une inhibition totale serait sans doute nécessaire [51] mais semble toutefois peu compatible avec la préservation importante de la myristoylation des protéines de la cellule infectée. Dans ce contexte physiopathologique, il existe une forte compétition pour la myristoylation entre les protéines de la cellule et les protéines virales. On estime que la myristoylation des protéines gag nécessiterait une concentration en C14:0 de l'ordre de
MA

MA

MA

Figure 5. Myristoylation de la protéine virale gag et régulations du cycle viral. 1. La protéine gag est synthétisée sous forme d'un précurseur polyprotéique Pr55gag (MA : matrice; CA : capside; NC : nucléocapside et p6) qui est myristoylé dans sa partie amino-terminale. Il est alors associé à la membrane [44]. 2. Une fois clivée du précurseur, la protéine MA séquestre l'acide myristique ( $40 \%$ du C14:0 reste exposé) ce qui ne permet plus son ancrage à la membrane. 3. Au cours du cycle viral, les protéines MA, en concentration plus importante, forment des trimères qui vont alors réexposer l'acide myristique et pouvoir être de nouveau adressées à la membrane. 
$1 \mu \mathrm{M}$ [21] alors qu'elle est d'environ $5 \mathrm{nM}$ [14] dans la cellule saine. Une meilleure connaissance de la spécificité des substrats des différentes isoformes de NMT est donc cruciale pour la poursuite de ces études d'inhibition.

\section{Conclusion}

Cette revue démontre, par les exemples choisis, les spécificités de régulation et de signalisation de l'acide myristique comparées à celles des autres acides gras. L'importance de la myristoylation pour assurer les fonctions des protéines concernées, la forte affinité de la NMT pour le substrat myristoyl-CoA, et enfin la faible teneur en acide myristique intracellulaire suggèrent que les apports exogènes en acide myristique, via l'alimentation, pourraient permettre de réguler de nombreux mécanismes cellulaires. Les recherches sur la relation entre disponibilité et fonctions métaboliques de l'acide myristique dans la cellule sont nécessaires à la définition d'éventuelles recommandations pour un apport alimentaire chez l'homme. $\diamond$

\section{SUMMARY}

\section{New regulatory and signal functions for myristic acid}

Myristic acid is a 14 carbon saturated fatty acid, which is mostly found in milk fat. In industrialized countries, its excessive consumption is correlated with an increase in plasma cholesterol and mortality due to cardiovascular diseases. Nevertheless, one feature of this fatty acid is its ability to acylate proteins, a reaction which is called $\mathrm{N}$-terminal myristoylation. This article describes various examples of important cellular regulations where the intervention of myristic acid is proven. Modulations of the cellular concentration of this fatty acid and its associated myristoylation function might be used as regulators of these metabolic pathways. $\diamond$

\section{RéFÉRENCES}

1. Katan MB, Zock PL, Mensink RP. Effects of fats and fatty acids on blood lipids in humans: an overview. Am J Clin Nutr $1994 ; 60$ : S1017-22.

2. Gnädig S, Chardigny JM, Sébédio JL. Lipides. In : Lait, Nutrition et Santé. Paris : Lavoisier Éditions, 2001.

3. Rioux V, Catheline D, Legrand P. In rat hepatocytes, myristic acid occurs through lipogenesis, palmitic shortening and lauric acid elongation. Animal $2007 ; 1: 820-6$.

4. Rioux V, Lemarchal P, Legrand P. Myristic acid, unlike palmitic acid, is rapidly metabolized in cultured rat hepatocytes. J Nutr Biochem 2000 ; 11 : 198-207.

5. Mensink RP, Katan MB. Effect of dietary fatty acids on serum lipids and lipoproteins. A metaanalysis of 27 trials. Arterioscler Thromb $1992 ; 12$ : $911-9$.

6. Tholstrup T, Vessby B, Sandstrom B. Difference in effect of myristic and stearic acid on plasma HDL cholesterol within 24 h in young men. Eur J Clin Nutr 2003; 57 : 735-42.

7. Rioux V, Catheline D, Bouriel M, Legrand P. Dietary myristic acid at physiologically relevant levels increases the tissue content of $C 20: 5 n-3$ and C20:3 n-6 in the rat. Reprod Nutr Dev $2005 ; 45: 599-612$.

8. Johnson DR, Bhatnagar RS, Knoll LJ, Gordon Jl. Genetic and biochemical studies of protein N-myristoylation. Annu Rev Biochem 1994 ; 63 : 869-914.

9. Giang DK, Cravatt BF. A second mammalian N-myristoyltransferase. J Biol Chem 1998 ; $273: 6595-8$

10. Mcllhinney RA, Young K, Egerton M, et al. Characterization of human and rat brain myristoylCoA:protein N-myristoyltransferase: evidence for an alternative splice variant of the enzyme. Biochem J $1998 ; 333: 491-5$.

11. Rioux V, Beauchamp $\varepsilon$, Pedrono F, et al. Identification and characterization of recombinant and native rat myristoyl-CoA: protein N-myristoyltransferases. Mol Cell Biochem 2006; $286: 161-70$
12. Kishore NS, Lu TB, Knoll LJ, et al. The substrate specificity of Saccharomyces cerevisiae myristoyl-CoA:protein N-myristoyltransferase. Analysis of myristic acid analogs containing oxygen, sulfur, double bonds, triple bonds, and/or an aromatic residue. J Biol Chem 1991; $266: 8835-55$.

13. Bhatnagar RS, Futterer K, Waksman G, Gordon JI. The structure of myristoyl-CoA:protein N-myristoyltransferase. Biochim Biophys Acta $1999 ; 1441: 162-72$.

14. Faergeman NJ, Knudsen J. Role of long-chain fatty acyl-CoA esters in the regulation of metabolism and in cell signalling. Biochem J 1997 ; $323: 1-12$.

15. Rioux V, Galat A, Jan G, et al. Exogenous myristic acid acylates proteins in cultured rat hepatocytes. J Nutr Biochem 2002; 13: 66-74.

16. Duronio RJ, Rudnick DA, Johnson RL, et al. Myristic acid auxotrophy caused by mutation of $S$. cerevisiae myristoyl-CoA:protein N-myristoyltransferase. J Cell Biol 1991; 113 : 1313-30.

17. Kokame K, Fukada Y, Yoshizawa T, et al. Lipid modification at the $\mathrm{N}$ terminus of photoreceptor G-protein alpha-subunit. Nature 1992 ; $359: 749-52$.

18. DeMar JC Jr, Anderson RE. Identification and quantitation of the fatty acids composing the $\mathrm{CoA}$ ester pool of bovine retina, heart, and liver. J Biol Chem 1997 ; 272 : 31362-8.

19. Zha J, Weiler S, Oh KJ, et al. Posttranslational N-myristoylation of BID as a molecular switch for targeting mitochondria and apoptosis. Science $2000 ; 290: 1761-5$

20. Maurer-Stroh S, Gouda M, Novatchkova M, et al. MyRbase: analysis of genome-wide glycine myristoylation enlarges the functional spectrum of eukaryotic myristoylated proteins. Genome Biol 2004 ; 5 : R21.

21. Boutin JA. Myristoylation. Cell Signal $1997 ; 9$ : 15-35.

22. Ntwasa M, Aapies S, Schiffmann DA, Gay NJ. Drosophila embryos lacking $\mathrm{N}$-myristoyltransferase have multiple developmental defects. Exp Cell Res $2001 ; 262$ : 134-44.

23. Yang SH, Shrivastav A, Kosinski C, et al. $\mathrm{N}$-myristoyltransferase $\mathrm{l}$ is essential in early mouse development. J Biol Chem $2005 ; 280: 18990-5$.

24. Sennequier N, Vadon-Le Goff S. Biosynthèse de monoxyde d'azote (NO) : mécanisme, régulation et contrôle. Med Sci (Paris) 1998 ; 14 : 1185-95.

25. Shaul PW, Smart EJ, Robinson LJ, et al. Acylation targets endothelia nitric-oxide synthase to plasmalemmal caveolae. J Biol Chem 1996 ; $271: 6518-22$.

26. Busconi L, Michel T. Endothelial nitric oxide synthase. $\mathrm{N}$-terminal myristoylation determines subcellular localization. J Biol Chem 1993; $268: 8410-3$

27. Robinson LJ, Busconi L, Michel T. Agonist-modulated palmitoylation of endothelial nitric oxide synthase. J Biol Chem 1995 ; 270 : 995-8.

28. Gonzalez $\varepsilon$, Kou R, Lin AJ, et al. Subcellular targeting and agonistinduced site-specific phosphorylation of endothelial nitric-oxide synthase. J Biol Chem $2002 ; 277$ : 39554-60.

29. Zhu W, Smart EJ. Myristic acid stimulates endothelial nitric-oxide synthase in a CD36- and an AMP kinase-dependent manner. J Biol Chem $2005 ; 280: 29543-50$.

30. Laugerette F, Passilly-Degrace P, Patris B, Niot I, Montmayeur JP, Besnard P. CD36, un sérieux jalon sur la piste du goût du gras. Med Sci (Paris) $2006 ; 22: 357-9$.

31. Isenberg JS, Jia Y, Fukuyama J, et al. Thrombospondin-1 inhibits nitric oxide signaling via CD36 by inhibiting myristic acid uptake. J Biol Chem $2007 ; 282$ : 15404-15.

32. Gong M, Wilson M, Kelly $T$, et al. HDL-associated estradiol stimulates endothelial NO synthase and vasodilation in an SR-BI-dependent manner. J Clin Invest 2003 ; 111 : 1579-87.

33. White J, Guerin T, Swanson H, et al. Diabetic HDL-associated myristic acid inhibits acetylcholine-induced nitric oxide generation by preventing the association of endothelial nitric oxide synthase with calmodulin. Am J Physiol Cell Physiol 2008; 294 : C295-305.

34. Harris WS, Von Schacky C. The omega-3 index: a new risk factor for death from coronary heart disease? Prev Med 2004 ; 39 : 212-20.

35. Bourre JM. Enrichissement de l'alimentation des animaux avec les acides gras omega-3: impact sur la valeur nutritionnelle de leurs produits pour l'homme. Med Sci (Paris) $2005 ; 21: 773-9$.

36. Jan S, Guillou H, D’Andrea S, et al. Myristic acid increases delta6desaturase activity in cultured rat hepatocytes. Reprod Nutr Dev 2004 ; $44: 131-40$.

37. Rioux V, Catheline D, Beauchamp $\varepsilon$, et al. Substitution of dietary oleic for myristic acid increases the tissue storage of alfa-linolenic acid and 
the concentration of docosahexaenoic acid in brain, red blood cells and plama in the rat. Animal $2008 ; 2: 636-44$.

38. Porta N, Auvin S. Acides gras polyinsaturés: propriétés et mécanismes anticonvulsivants. Med Sci (Paris) 2009 ; 25 : 51-6.

39. Dabadie $H$, Peuchant $\varepsilon$, Bernard $M$, et al. Moderate intake of myristic acid in sn-2 position has beneficial lipidic effects and enhances DHA of cholesteryl esters in an interventional study. J Nutr Biochem 2005; $16: 375-82$.

40. Beauchamp $\varepsilon$, Goenaga D, Le Bloc'h J, et al. Myristic acid increases the activity of dihydroceramide Delta4-desaturase 1 through its $\mathrm{N}$-terminal myristoylation. Biochimie 2007 ; 89 : 1553-61.

41. Borgese $\mathrm{N}$, Aggujaro D, Carrera $\mathrm{P}$, et al. A role for $\mathrm{N}$-myristoylation in protein targeting: $\mathrm{NADH}$-cytochrome b5 reductase requires myristic acid for association with outer mitochondrial but not $\varepsilon R$ membranes. J Cell Biol 1996; $135: 1501-13$

42. Stiban J, Fistere D, Colombini M. Dihydroceramide hinders ceramide channel formation: implications on apoptosis. Apoptosis 2006 ; $11: 773-80$.

43. Corbin A, Grigorov B, Roingeard P, et al. Une nouvelle vision de l'assemblage du VIH-1. Med Sci (Paris) $2008 ; 24: 49-55$

44. Henderson $L \varepsilon$, Bowers MA, Sowder RC $2^{\text {nd }}$, et al. Gag proteins of the highly replicative $M N$ strain of human immunodeficiency virus type 1 : posttranslational modifications, proteolytic processings, and complete amino acid sequences. J Virol $1992 ; 66: 1856-65$.
45. Schultz AM, Oroszlan S. In vivo modification of retroviral gag gene-encoded polyproteins by myristic acid. J Virol $1983 ; 46: 355-61$.

46. Takamune N, Gota K, Misumi S, et al. HIV-1 production is specifically associated with human NMTl long form in human NMT isozymes. Microbes Infect $2008 ; 10: 143-50$.

47. Seaton KE, Smith CD. N-Myristoyltransferase isozymes exhibit differential specificity for human immunodeficiency virus type 1 Gag and Nef. J Gen Virol 2008 ; 89 : 288-96.

48. Spearman P, Horton R, Ratner L, Kuli-Zade I. Membrane binding of human immunodeficiency virus type 1 matrix protein in vivo supports a conformational myristyl switch mechanism. J Virol $1997 ; 71: 6582-92$.

49. Boutin JA. La N-myristoyl transférase, carrefour entre virologie et oncologie: une voie d'accès à des anticancéreux et à des antiviraux d'un genre nouveau? Med Sci (Paris) 1993 ; $9: 684-92$

50. Lindwasser OW, Resh MD. Myristoylation as a target for inhibiting HIV assembly: unsaturated fatty acids block viral budding. Proc Natl Acad Sci USA 2002 ; 99 : 13037-42.

51. Morikawa $\mathrm{Y}$, Hinata $\mathrm{S}$, Tomoda $\mathrm{H}$, et al. Complete inhibition of human immunodeficiency virus Gag myristoylation is necessary for inhibition of particle budding. J Biol Chem 1996 ; $271: 2868-73$.

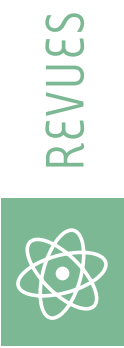

\section{TIRÉS À PART}

V. Rioux

\section{Glomax Multi Détection lumineuse simplicité}
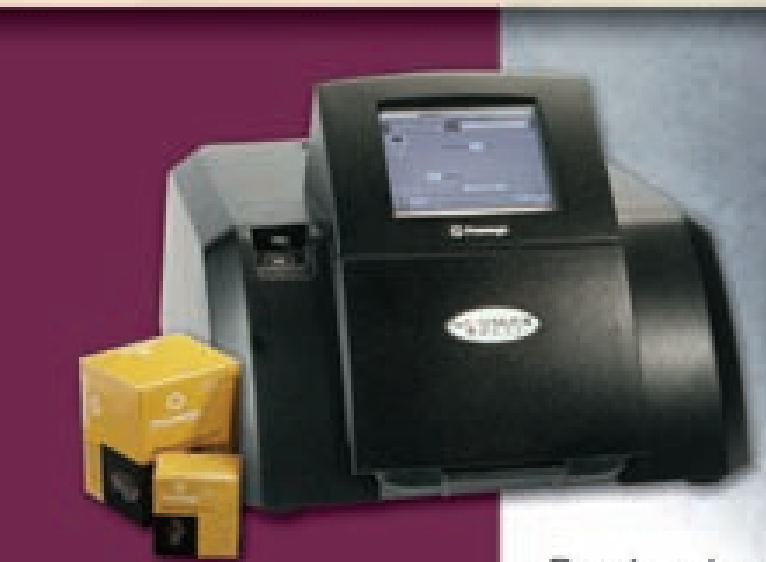

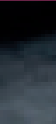

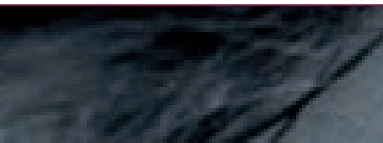

prong

\section{Rendons la performance accessible}

- Deftection luminescence. absorbanco, flucresconce.

- Applications nombrouses : gane rapportaur, toocicolopie. viabilite collukire.

- Ben actiost pour la nocherche. notamment en cancleciogio of

biologio celubire.

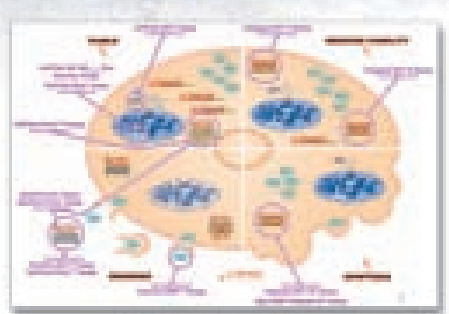

service client

ONavat 0000487999 